\title{
NUMERICAL INVESTIGATION OF NONLINEAR WAVES CONNECTED TO BLOOD FLOW IN AN ELASTIC TUBE WITH VARIABLE RADIUS*
}

\author{
Zlatinka I. Dimitrova \\ "G. Nadjakov" Insitute of Solid State Physics," \\ 72, Tzarigradsko Chaussee Blvd., 1784 Sofia, Bulgaria, \\ e-mail: zdim@issp.bas.bg
}

[Received 23 September 2015. Accepted 16 November 2015]

\begin{abstract}
We investigate flow of incompressible fluid in a cylindrical tube with elastic walls. The radius of the tube may change along its length. The discussed problem is connected to the fluid-structure interaction in large human arteries and especially to nonlinear effects. The long-wave approximation is applied to solve model equations. The obtained model Korteweg-deVries equation possessing a variable coefficient is reduced to a nonlinear dynamical system of three first order differential equations. The low probability of a solitary wave arising is shown. Periodic wave solutions of the model system of equations are studied and it is shown that the waves, that are consequence of the irregular heart pulsations may be modelled by a sequence of parts of such periodic wave solutions.
\end{abstract}

KEY WORDS: Blood flow in large arteries, long-wave approximation, variable coefficient Korteweg-deVries equation, periodic solutions.

\section{Introduction}

Nonlinear phenomena are usual for fluid mechanics [1]-[5]. One of the most interesting nonlinear phenomena is the nonlinear waves propagating in various media [6]-[9] and especially in fluids $[10,11]$. In this paper, we shall discuss nonlinear waves arising do to fluid-structure interaction in large arteries [12]-[14]. There exist many kinds of possible methodologies to investigate traveling waves of the model nonlinear partial differential equations. One of them is

\footnotetext{
*This research was supported partially by the project FNI I 02/3 "Computer modeling and clinical study of arterial aneurysms of humans".
} 
to reduce the corresponding nonlinear partial differential equation (PDE) to a system of nonlinear ordinary differential equations and then to investigate the obtained system numerically. Another one is to apply methods for obtaining (exact) solutions of the studied nonlinear partial differential equation [15]-[19]. We shall use the first of the above methodologies in this paper.

Fluid flow connected to spreading of pressure pulses in elastic tubes is of interest for large arteries arterial mechanics [20]-[26]. In this case, the nonlinearities are important for the study of the flow and because of this, one has to use non-linear model differential equations. In the mathematical models, arteries usually are treated as circularly cylindrical long homogeneous isotropic tubes, which length is much larger than its radius (i.e. the corresponding tube can be considered as thin tube with respect to the ratio length/thickness). Below, we shall study the propagation of non-linear waves in a fluid-filled long elastic tube with variable radius. The fluid will be incompressible (this is a reasonable assumption for the case of blood flow in large arteries) and the tube will be isotropic, inhomogeneous and prestressed. The model equations of the motion of the tube wall will be reduced to a variant of the Korteweg-deVries equation with one variable coefficient. This nonlinear partial differential equation will be further reduced to a system of three nonlinear ordinary differential equations for the case of traveling waves. The system of nonlinear ordinary differential equations will be studied numerically.

The organization of the paper is as follows. In Section 2 we discuss the model equations for the blood flow in a large artery. In Section 3, by application of the long-wave approximation the model equation will be reduced to a forced Korteweg-deVries equation and this equation will be further reduced to a system of three nonlinear ordinary differential equation. In Section 4 we perform a numerical study of the system of nonlinear ODEs. Several concluding remarks are summarized in Section 5.

\section{Mathematical formulation of the model}

For our study, we shall use the nonlinear model presented in [20]. The model has two parts: equations of the elastic tube and equation of the fluid in the tube. First, we describe the model equations of the elastic tube. We set a cylindrical polar co-ordinate system with axial axis coinciding to the axis of the (straight) studied tube. We denote the base vectors of this co-ordinate system 
as $\vec{e}_{r}, \vec{e}_{\theta}, \vec{e}_{z} . R_{0}$ is the radius of the tube before the stretching. The tube is assumed to be tapered (with small tapering angle $\Phi$ ). $Z^{*}$ is the axial co-ordinate along the axis of the elastic tube. Because of the tapering, the radial coordinate of the tube point with axial co-ordinate $Z$ will be $R_{0}+Z^{*} \sin \Phi \approx R_{0}+Z^{*} \Phi$. Thus, the initial coordinate of the point will be $\vec{R}=\left(R_{0}+Z^{*} \Phi\right) \vec{e}_{r}+Z^{*} \vec{e}_{z}$.

We assume that there is axial stretch of the tube. After the stretching the axial co-ordinate becomes $z^{*}=\lambda_{z} Z^{*}$, where $\lambda_{z}$ is the axial stretch ratio (for the case without stretching $\lambda_{z}=1$ ). In addition, there is axially-dependent static pressure $P_{0}\left(Z^{*}\right)$, imposed on the tube (it has to be understood as the end of the diastolic pressure). Let $r_{0}$ be the deformed radius of the tube at the origin of the coordinate system. We have to introduce the function $f^{*}(z)$, which characterizes the radius change, if the form of the tube doesn't remain cone. Additional (dynamical) displacement of the tube arises from the presence of fluid (blood) flow. This displacement depends on the coordinate $z^{*}$ and on the time $t^{*}$. Let us denote this displacement as $u^{*}\left(z^{*}, t^{*}\right)$. Then, the coordinate of the point of the tube becomes:

$$
\vec{r}_{0}\left(z^{*}\right)=\left[r_{0}+f\left(z^{*}\right)+u^{*}\left(z^{*}, t^{*}\right)\right] \vec{e}_{r}+z^{*} \vec{e}_{z} .
$$

In this case, the lengths of the elementary meridional and circumferential tubes are as follows. The arc-length of the meridional curve element is:

$$
d s_{z}^{0}=\left[1+\left(\frac{\partial f^{*}}{\partial z^{*}}+\frac{\partial u^{*}}{\partial z^{*}}\right)^{2}\right]^{1 / 2} d z^{*}
$$

The arc-length of the circumferential curve element is:

$$
d s_{\theta}^{0}=\left(r_{0}+f^{*}+u^{*}\right) d \theta .
$$

The corresponding stretch ratios after the static and dynamic deformation (when the tube does not remain cone after the static deformation) are:

$$
\lambda_{1}=\lambda_{z} \frac{\left[\left(1+\left[\left(\partial f^{*} / \partial z^{*}\right)+\left(\partial u^{*} / \partial z^{*}\right)\right]^{2}\right]^{1 / 2}\right.}{\left(1+\Phi^{2}\right)^{1 / 2}} ; \quad \lambda_{2}=\lambda_{z} \frac{r_{0}+f^{*}+u^{*}}{\lambda_{z} R_{0}+z^{*} \Phi} .
$$

For the case when $\Phi=0$ (i.e. the tube before applying the static pressure is cylinder and not a cone): $\lambda_{1}=\lambda_{z}\left[\left(1+\left[\left(\partial f^{*} / \partial z^{*}\right)+\left(\partial u^{*} / \partial z^{*}\right)^{2}\right]^{1 / 2}\right] ; \lambda_{2}=\right.$ $\lambda_{z} \frac{r_{0}+f^{*}+u^{*}}{\lambda_{z} R_{0}}$. The relationship for $\lambda_{2}$ can be written as follows $\left(\lambda_{\theta}=r_{0} / R_{0}\right)$ : 
$\lambda_{2}=\frac{r_{0}}{R_{0}}+\frac{f^{*}+u^{*}}{R_{0}}=\lambda_{\theta}+\frac{f^{*}+u^{*}}{R_{0}}$. In the case, when the tube is deformed and in the general case the unit normal vector $\vec{n}$ does not coincide to the unit vector $\vec{e}_{r}$. The unit tangential vector $\vec{t}$ of the curved surface also does not coincide to the vector $\vec{e}_{z}$. The unit normal and tangential vectors can be expressed by the unit vectors $\vec{e}_{r}, \vec{e}_{z}$. When we take into account, that:

$$
\left[\left(1+\left[\left(\partial f^{*} / \partial z^{*}\right)+\left(\partial u^{*} / \partial z^{*}\right)\right]^{2}\right]^{1 / 2}=\frac{\lambda_{1}}{\lambda_{z}},\right.
$$

then:

$$
\begin{aligned}
\vec{t} & =\frac{\left[\left(\partial f^{*} / \partial z^{*}\right)+\left(\partial u^{*} / \partial z^{*}\right)\right] \vec{e}_{r}+\vec{e}_{z}}{\left(1+\left[\left(\partial f^{*} / \partial z^{*}\right)+\left(\partial u^{*} / \partial z^{*}\right)\right]^{2}\right)^{1 / 2}} \\
& =\frac{\lambda_{z}}{\lambda_{1}}\left(\left[\left(\partial f^{*} / \partial z^{*}\right)+\left(\partial u^{*} / \partial z^{*}\right)\right] \vec{e}_{r}+\vec{e}_{z}\right), \\
\vec{n} & =\frac{\vec{e}_{r}-\left[\left(\partial f^{*} / \partial z^{*}\right)+\left(\partial u^{*} / \partial z^{*}\right)\right] \vec{e}_{z}}{\left(1+\left[\left(\partial f^{*} / \partial z^{*}\right)+\left(\partial u^{*} / \partial z^{*}\right)\right]^{2}\right)^{1 / 2}} \\
& =\frac{\lambda_{z}}{\lambda_{1}}\left[\vec{e}_{r}-\left[\left(\partial f^{*} / \partial z^{*}\right)+\left(\partial u^{*} / \partial z^{*}\right)\right] \vec{e}_{z}\right] .
\end{aligned}
$$

The forces responsible for the motion of a fluid element are: the force of movement in radial direction due to the existing pressure difference; the force along the meridional curve; the force acing along the circumferential curve. The force connected to the motion of the tube element is equal to the mass of the tube element multiplied by its acceleration. Let the tube thickness before the static deformation is $H$. The thickness of the tube after the static deformation will be $h$. Then, the mass of the tube element is approximately $\rho_{w} h R d \theta d z$, where $\rho_{w}$ is the mass density of the material of the tube. The acceleration of the element is equal to $\frac{\partial^{2} u^{*}}{\partial t^{* 2}}$. Thus, this term of the equation of balance of forces becomes $\rho_{w} h R d \theta d z \frac{\partial^{2} u^{*}}{\partial t^{* 2}}$. The tube thickness $h$ after the static deformation can be expressed by the tube thickness $H$ before the static deformation. The assumption is that the material is incompressible. This leads to $\lambda_{1} \lambda_{2} h=H \rightarrow h=\frac{H}{\lambda_{1} \lambda_{2}}$. In our case (when axial stretching exists) the initial radius $R_{0}$ for tube with length $d l$ transforms to radius $R$ for a tube 
of length $\lambda_{z} d l$. Assuming that the area remains the same, we have $R=R_{0} / \lambda_{z}$. Thus, for the force per unit $d \theta d z$ we obtain $\rho_{w} H\left(R_{0} / \lambda_{z}\right) \frac{\partial^{2} u^{*}}{\partial t^{* 2}}$.

The pressure force, that acts on the tube element, is equal to the pressure difference $P-P_{e}$ (where $P$ is the pressure in the tube and $P_{e}$ is the external pressure) multiplied by the surface of the tube element that is $R d \theta d z$. Then, this force becomes $\left(P-P_{e}\right) \cos \phi(R d \theta d l)$. In our case $P_{e}=P_{0}$ and $P-P_{e}=P^{*}$. The co-ordinate $R$ connected to the element of the tube is $R=r_{0}+f^{*}+u^{*}$. And the entire force per unit $d \theta d z$ becomes $P^{*}\left(r_{0}+f^{*}+u^{*}\right)$.

The remaining two forces are connected to the membrane forces, that act along the circumferential and meridional curves of the tube. For unit $d \theta d z$, the force acting along the meridional curve is $F_{2} \vec{t}$. Its vertical component is $T_{2}=F_{2} \vec{t} \cdot \vec{n}$ from where $T_{2}=-F_{2} \lambda_{z} / \lambda_{1}$ and $F_{2}=-T_{2} \lambda_{1} / \lambda_{z}$. The force $F_{1}$ is $[20,27]: F_{1}=\frac{\partial}{\partial z^{*}}\left[\frac{\lambda_{z}}{\lambda_{1}}\left(r_{0}+f^{*}+u^{*}\right)\left(\frac{\partial f^{*}}{\partial z}+\frac{\partial u^{*}}{\partial z}\right) T_{1}\right]$. The balance of the above forces is:

$$
\begin{array}{r}
-\frac{\lambda_{1}}{\lambda_{z}} T_{2}+\frac{\partial}{\partial z^{*}}\left\{\frac{\lambda_{z}\left(r_{0}+f^{*}+u^{*}\right)\left(\frac{\partial f^{*}}{\partial z^{*}}+\frac{\partial u^{*}}{\partial z^{*}}\right)}{\lambda_{1}} T_{1}\right\}+ \\
P^{*}\left(r_{0}+f^{*}+u^{*}\right)=\rho_{w} H \frac{R_{0}}{\lambda_{z}} \frac{\partial^{2} u^{*}}{\partial t^{* 2}} .
\end{array}
$$

Let $\mu\left(z^{*}\right)$ be the variable shear modulus of the tube material and $\Pi$ be the strain energy density function of the membrane. Then, the wall tensions $T_{1,2}$ can be written as:

$$
T_{1}=\frac{H}{\lambda_{2}} \mu\left(z^{*}\right) \frac{\partial \Pi}{\partial \lambda_{1}} ; \quad T_{2}=\frac{H}{\lambda_{1}} \mu\left(z^{*}\right) \frac{\partial \Pi}{\partial \lambda_{2}} .
$$

After a substitution of Eq.(9) in Eq. (8) we obtain the pressure $P^{*}$ as a function of $u^{*}$ and its derivatives:

$$
\begin{aligned}
P^{*}= & \frac{\rho_{w} H R_{0}}{\lambda_{z}\left(r_{0}+f^{*}+u^{*}\right)} \frac{\partial^{2} u^{*}}{\partial t^{* 2}}+\frac{\mu\left(z^{*}\right) H}{\lambda_{z}\left(r_{0}+f^{*}+u^{*}\right)} \frac{\partial \Pi}{\partial \lambda_{2}}- \\
& \frac{\lambda_{z} R_{0} H}{\left(r_{0}+f^{*}+u^{*}\right)} \frac{\partial}{\partial z^{*}}\left[\frac{\mu\left(z^{*}\right)}{\lambda_{1}}\left(\frac{\partial f^{*}}{\partial z^{*}}+\frac{\partial u^{*}}{\partial z^{*}}\right) \frac{\partial \Pi}{\partial \lambda_{1}}\right]
\end{aligned}
$$

The model equations of the fluid motion in the tube are as follows. The blood in a large arteries can be approximated by a Newtonian fluid with respect to its flow (this is not the case for blood flow in small arteries). In addition, the 
viscosity of the blood may be neglected as a first approximation $[28,29]$ and the variation of the quantities with the radial coordinate will be disregarded, too. So, the Euler equations in cylindrical coordinates is

$$
\frac{\partial w^{*}}{\partial t^{*}}+w^{*} \frac{\partial w^{*}}{\partial z^{*}}+\frac{1}{\rho_{f}} \frac{\partial P^{*}}{\partial z^{*}}=0,
$$

where $\rho_{f}$ is the density of the fluid and $w^{*}$ is the axial fluid velocity. In addition $\frac{\partial A^{*}}{\partial t^{*}}+\frac{\partial}{\partial z^{*}}\left(w^{*} A^{*}\right)=0$, where $A^{*}$ is the cross-sectional area of the tube. This area is $A^{*}=\pi\left(r_{0}+f^{*}+u^{*}\right)^{2}$.

\section{Non-dimensionalization of the equations and long-wave ap- proximation}

The following non-dimensional quantities $t, z, R_{0}, u, w, m, p, c_{0}$ and $E(z)$ are introduced as follows: $t^{*}=t\left(\frac{R_{0}}{c_{0}}\right) ; c_{0}^{2}=\frac{\mu_{0} H}{\rho_{f} R_{0}} ; z^{*}=R_{o} z ; r_{0}=\lambda_{\theta} R_{0}$; $u^{*}=R_{0} u ; m=\frac{\rho_{w} H}{\rho_{f} R_{0}} ; w^{*}=w c_{0} ; P^{*}=p \rho_{f} c_{0}^{2} ; \mu=\mu_{0} E(z)$. The model equations for the unknown functions $u, w$ and $p$ in non-dimensionl coordinates are:

$$
\begin{gathered}
\frac{\partial w}{\partial t}+w \frac{\partial w}{\partial z}+\frac{\partial p}{\partial z}=0 \\
2 \frac{\partial u}{\partial t}+2 w\left(\frac{\partial f}{\partial z}+\frac{\partial u}{\partial z}\right)+\left(\lambda_{\theta}+f+u\right) \frac{\partial w}{\partial z}=0, \\
p=\frac{m}{\lambda_{z}\left(\lambda_{\theta}+f+u\right)} \frac{\partial^{2} u}{\partial t^{2}}+\frac{E(z)}{\lambda_{z}\left(\lambda_{\theta}+f+u\right)} \frac{\partial \Pi}{\partial \lambda_{2}}- \\
\frac{\lambda_{z}}{\left(\lambda_{\theta}+f+u\right)} \frac{\partial}{\partial z}\left[\frac{E(z)\left(\frac{\partial f}{\partial z}+\frac{\partial u}{\partial z}\right)}{\lambda_{1}} \frac{\partial \Pi}{\partial \lambda_{1}}\right] .
\end{gathered}
$$

In order to proceed further, we shall consider the case of propagation of small (but finite) amplitude waves in an inhomogeneous thin elastic tube with variable radius and filled with Newtonian fluid. The following stretched coordinates $\xi=$ $\epsilon^{1 / 2}(z-g t) ; \tau=\epsilon^{3 / 2} z$, can be introduced, using small parameter $\epsilon$ describing weakness of nonlinearity. From here, $z=\tau \epsilon^{-2 / 3}$ and we can use the notations $h(\epsilon, \tau)=f(z)$ and $\widehat{E}(\tau, \epsilon)=E(z)$. 
Next, we expand $u, w, p, h$ and $\widehat{E}$ in series of the small parameter $\epsilon$ :

$$
\begin{aligned}
& u=\epsilon u_{1}(\xi, \tau)+\epsilon^{2} u_{2}(\xi, \tau)+\ldots ; w=\epsilon w_{1}(\xi, \tau)+\epsilon^{2} w_{2}(\xi, \tau)+\ldots, \\
& p=p_{0}+\epsilon p_{1}(\xi, \tau)+\epsilon^{2} p_{2}(\xi, \tau)+\ldots ; h(\epsilon, \tau)=1+\epsilon h_{1}(\tau)+\ldots,
\end{aligned}
$$

(15) $\widehat{E}(\epsilon, \tau)=1+\epsilon E_{1}(\tau)+\ldots$

Let $U(\xi, \tau)=u_{1}$. From the systems of equations of orders $\epsilon$ and $\epsilon^{2}$, we obtain for $U$ the partial differential equation:

$$
\frac{\partial U}{\partial \tau}+\mu_{1} U \frac{\partial U}{\partial \xi}+\mu_{2}(\tau) \frac{\partial U}{\partial \xi}+\mu_{3} \frac{\partial^{3} U}{\partial \xi^{3}}=0 .
$$

The other unknown functions and coefficients are:

$$
\begin{aligned}
w_{1} & =2 \frac{g}{\lambda_{0}}\left[U-\left(h_{1}+\frac{\beta_{0}}{\beta_{1}} E_{1}\right)\right] ; \beta_{0}=\left.\frac{1}{\lambda_{\theta} \lambda_{z}} \frac{\partial \Pi}{\partial \lambda_{\theta}}\right|_{u=0} ; \\
\beta_{1} & =\left.\frac{1}{\lambda_{\theta} \lambda_{z}} \frac{\partial^{2} \Pi}{\partial \lambda_{\theta}^{2}}\right|_{u=0}-\frac{\beta_{0}}{\lambda_{\theta}} ; \beta_{2}=\left.\frac{1}{2 \lambda_{\theta} \lambda_{z}} \frac{\partial^{3} \Pi}{\partial \lambda_{\theta}^{3}}\right|_{u=0}+\frac{\beta_{0}}{\lambda_{\theta}^{2}}-\frac{\beta_{1}}{\lambda_{\theta}} ; \\
\alpha_{0} & =\left.\lambda_{z} \frac{\partial \Pi}{\partial \lambda_{z}}\right|_{u=u} ; g^{2}=\frac{\beta_{1}}{2 \lambda_{\theta}} ; p_{1}=2 \frac{g^{2}}{\lambda_{\theta}}\left(h_{1}+U\right)+\beta_{0} E_{1},
\end{aligned}
$$

and $\mu_{1,2,3}$ are as follows:

$$
\begin{aligned}
& \mu_{1}=\frac{5}{2 \lambda_{\theta}}+\frac{\beta_{2}}{\beta_{1}} ; \quad \mu_{2}(\tau)=\left(\frac{\beta_{2}}{\beta_{1}}-\frac{3}{2 \lambda_{\theta}}\right) h_{1}(\theta)+\left(\frac{1}{2}-\frac{2 \beta_{0}}{\beta_{1} \lambda_{\theta}} E_{1}(\tau)\right) \\
& \mu_{3}=\frac{1}{\lambda_{z} \lambda_{\theta}}\left(\frac{m}{4 \lambda_{\theta}}-\frac{\alpha_{0}}{2 \beta_{1}}\right) .
\end{aligned}
$$

Finally, we have to deal with the variable coefficient $\mu_{2}(\theta)$ in Eq.(16). We introduce the new coordinate $\eta=\xi+\tau-\int_{0}^{\tau} d s \mu_{2}(s)$. The substitution of this coordinate in Eq. (16) leads to the equation:

$$
\frac{d U}{d \eta}+\mu_{1} U \frac{d U}{d \eta}+\mu_{3} \frac{d^{3} U}{d \eta^{3}}=0
$$

Let $V=d U / d \eta$ and $W=d V / d \eta$. Then, Eq. (19) is reduced to the following system of three equations for the unknown functions $U, V, E$ :

$$
\frac{d U}{d \eta}=V ; \quad \frac{d V}{d \eta}=W ; \quad \mu_{3} \frac{d W}{d \eta}=-V\left(1+\mu_{1} U\right) .
$$


The quantity $U$ is connected to the deformation of the tube, due to the presence of fluid with pulsate pressure and this quantity is the main quantity of interest for us in this paper. This is nondimensional displacement of the tube wall in the first approximation in specific coordinates $\eta$.

\section{Numerical results}

Eq. (16) possesses a solitary wave solution. This solitary wave solution is connected to the solitary wave solution of the classical Korteweg-deVries equation:

$$
\frac{\partial D}{\partial t}+\alpha D \frac{\partial D}{\partial x}+\beta \frac{\partial^{3} D}{\partial x^{3}}=0
$$

Now, let:

$$
D^{*}=\alpha D / 6 ; \quad x^{*}=x / \beta^{1 / 2} ; \quad t^{*}=t / \beta^{1 / 2} .
$$

The result of substitution of Eq.(22) in Eq.(21) (we drop the ${ }^{*}$-s) is:

$$
\frac{\partial D}{\partial t}+6 D \frac{\partial D}{\partial x}+\frac{\partial^{3} D}{\partial x^{3}}=0 .
$$

Let us search for travelling-wave solutions of Eq.(23) of the kind $D(x, t)=$ $D(\zeta)=D(x-v t)$. Eq.(23) becomes:

$$
\frac{d D}{d \zeta}-\frac{6}{v} D \frac{d D}{d \zeta}-\frac{1}{v} \frac{d^{3} D}{d \zeta^{3}}=0
$$

which is the same as Eq. (19), when $\mu_{1}=-6 / v$ and $\mu_{3}=-1 / v$. The solitary wave solution of Eq.(17) is:

$$
U(\eta)=\frac{v}{2} \operatorname{sech}^{2}\left(\frac{v^{1 / 2}}{2} \eta\right) .
$$

The realization of this solution for the case of wall displacement in large arteries, however, has low probability, because of two reasons. First, the existence of the solution (25) requires a relationship between $\mu_{1}$ and $\mu_{3}$ (namely $\mu_{1}=6 \mu_{3}$ ), that may not be present in the practical situations. And second, the realization 
of the solution (25) requires specific boundary conditions. The derivatives of (25) are as follows:

$$
\begin{array}{r}
\frac{d U}{d \eta}=-\frac{v^{3 / 2} \sinh \left(v^{1 / 2} \eta / 2\right)}{\cosh ^{3}\left(v^{1 / 2} \eta / 2\right)} ; \frac{d^{2} U}{d \eta^{2}}=\frac{v^{2}\left[2 \cosh ^{2}\left(v^{1 / 2} \eta / 2\right)-3\right]}{4 \cosh ^{4}\left(v^{1 / 2} \eta / 2\right)}, \\
\frac{d^{3} U}{d \eta^{3}}=-\frac{v^{5 / 2} \sinh \left(v^{1 / 2} \eta / 2\right)\left[\cosh ^{2}\left(v^{1 / 2} \eta / 2\right)-3\right]}{2 \cosh ^{5}\left(v^{1 / 2} \eta / 2\right)} ; \ldots
\end{array}
$$

Thus, the boundary conditions for realization of the solitary wave at $\eta=0$ should be:

$$
U(0)=\frac{v}{2} ;\left.\frac{d U}{d \eta}\right|_{\eta=0}=0 ;\left.\frac{d^{2} U}{d \eta^{2}}\right|_{\eta=0}=-\frac{v^{2}}{4} ;\left.\frac{d^{3} U}{d \eta^{3}}\right|_{\eta=0}=0 ; \ldots
$$

The realization of these boundary conditions is not very probable, as the heart pulsations are slightly irregular with respect to amplitude and time between the beats. Thus, if a solitary wave solution is realized for a pulsation the next pulsation will lead to slight change of the boundary conditions and the next solution will be not solitary wave. Then, another scenario for the wall displacement is more probable and this scenario is connected to the periodic solutions of Eq. (19).

The periodic wave solutions of Eq. (19) can be realized for much more values of the boundary conditions and for different amplitudes of quantity $U$. Several examples of periodic solutions of Eq. (19), obtained through the system of equations (20) are shown in Fig. 1:

The periodic solutions can be used for construction of the wave motion of the wall because of their larger parameter regions of existence in presence of blood flow from irregularity of heart beats as follows. Let the heart makes a pulsation and the blood pulse starts to propagate in the artery. This gives rise to displacement of arterial wall and the dynamical deformation of the wall can be modelled by a half a period of the periodic wave solution of Eq. (19). When the next pulsation comes, one can stop at the corresponding values of $U$ and its derivatives and can treat them as the new initial conditions. These new initial conditions lead to slightly different periodic solution. Half a period of this solution can be used to model the dynamical displacement up to the moment of the next pulsation. At this moment, the reached values of $U$ and its derivatives are again the initial conditions for next displacement arising from 

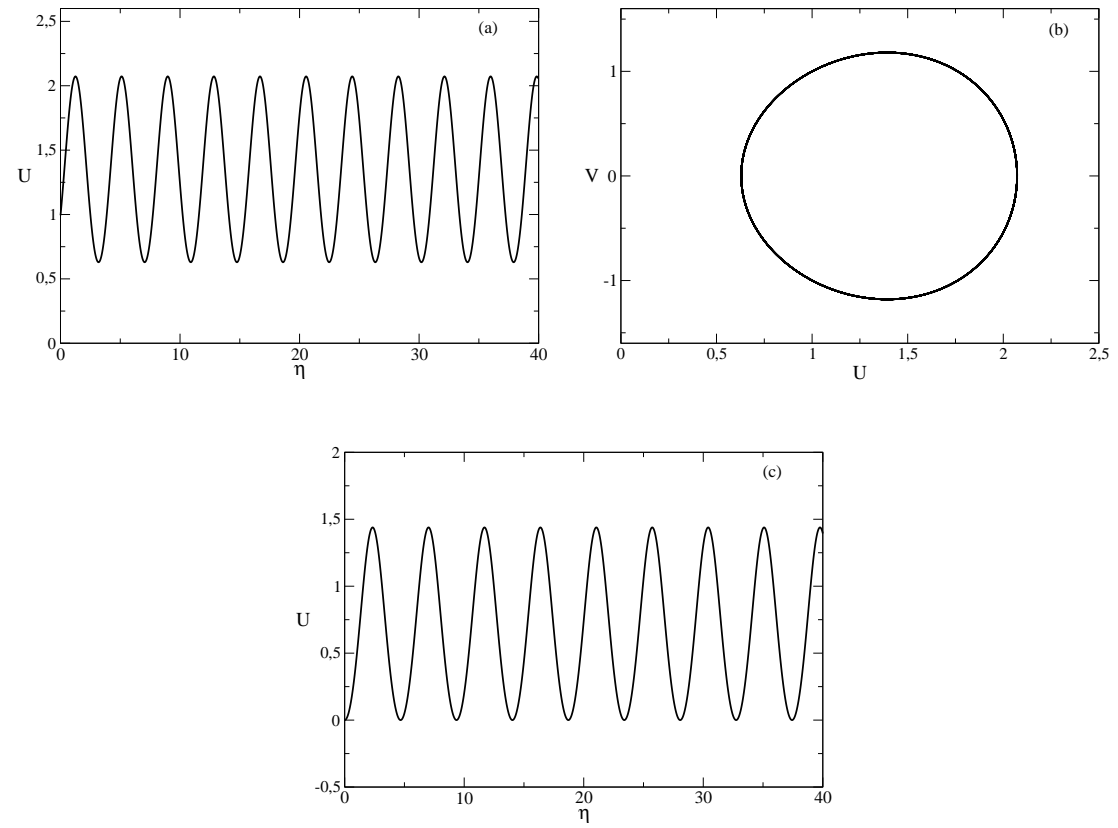

Fig. 1. Examples for periodic wave solutions of Eq. (20). Figure (a): $\mu_{1}=1.43 ; \mu_{3}=1.1 . U(0)=V(0)=W(0)=0.99$. Figure $(\mathrm{b})$ : Diagram of the components $U$ and $V$ for the solution with parameters and initial conditions, that are the same as those in Fig. (a). Figure (c): $\mu_{1}=$ 4.0; $\mu_{3}=2.1$. $U(0)=V(0)=0 ; W(0)=1$. One may observe that the period and the amplitude of this solution are different with respect to the period and the amplitude of the solution from Fig. (a)

the third pulsation, etc. In such a way, a sequence of slightly different wave solutions of model equation can describe dynamical deformation of arterial wall, cased by the slight irregularity of the heart pulsation (shown in Fig. 2.) It is well known, that the time intervals between the heart pulsations are long-range correlated and this is one of the numerous arising of long-range correlations in various systems [30]-[33]. Such long-range correlated pseudorandom sequences modelling heart activity may be generated by a computer program and the values in the sequence will determine the end of the corresponding wave train and the beginning of the next wave train. This was realized in Fig. 2. 


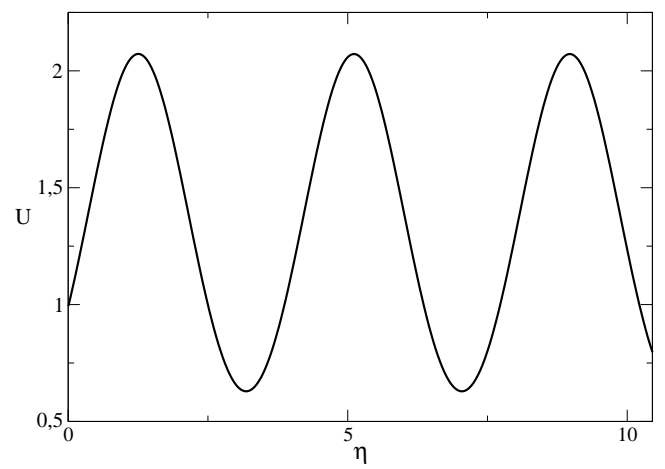

Fig. 2. Periodic behaviour of quantity $U$, represented the dynamical wall displacement in coordinate $\eta$, constructed by parts of periodic solutions of Eq. (19) by the algorithm described in the text. It may seem, that behaviour as a single wave is shown in the figure. Actually, the dynamical deformation is constructed by the parts of three different periodic solutions of Eq.(19). Namely, it is assumed that after the pulsation a wall displacement starts at $\eta=0$ with initial conditions $U(0)=V(0)=W(0)=0.99$ and $\mu_{1}=1.43 ; \mu_{3}=1.1$. The wave can reach a point with coordinate $\eta=2.844$, where initial conditions are $U(2.844)=0.7253 ; V(2.844)=-0.5571 ; W(2.844)=1.5255$ and $\mu_{1}=1.42 ; \mu_{3}=1.11$. The third place is at $\eta=6.924$ with initial conditions $U(6.924)=0.6409 ; V(6.924)=-0.2000 ; W(6.924)=1.6772$ and $\mu_{1}=1.44 ; \mu_{3}=1.08$

\section{Concluding remarks}

In this paper, we have shown that the area of research on blood flow is a large area for application of the methods of nonlinear dynamics. Even the relative single problem such as investigation of arterial wall displacement caused by pulsatile flow (where the fluid can be treated as Newtonian and the longwave approximation significantly simplifies the equations) lead to relatively complicated model equations, such as the discussed above variable coefficient $\mathrm{KdV}$ equation. We have stressed, that the solitary wave solution of the model equation requires very specific initial conditions and relationship among the two model parameters. Because of this, the probability for realization of this solution is small. We have discussed another kind of solutions of the nonlinear model equation, that are much more probable for realization: they do not require relationship between the two parameters of the model and are robust against change of the initial conditions, due to the irregularities of the pulsation dynamics of the heart. These solutions are the periodic solutions of the system of equations (20). Using parts of these solutions, one can construct a model 
profile of motion of arterial wall, that reflects the irregularities and the longrange correlations presented in the pulsation activity of human heart.

\section{REFERENCES}

[1] HariRi, K., S. A. O. Aliev. Fluid Mechanics and Heat Transfer, Advances in Nonlinear Dynamics Modeling. FL, Boca Raton, CRC Press, 2015.

[2] Yomosa, S. Solitary Waves in Large Blood Vessels. Journal of the Physical Society of Japan, 56 (1987), 506-520.

[3] Voltairas, P. A., D. I. Fotiadis, D. I. Massalas, L. K. Michalis. Anharmonic Analysis of Arterial Blood Pressure and Flow Pulses. Journal of Biomechanics, 38 (2005), 1423-1431.

[4] Vitanov, N. K., F. H. Busse. Bounds on the Heat Transport in a Horizontal Fluid Layer with Stress-free Boundaries. Zeitschrift für Angewandte Nathematik und Physik (ZAMP), 48 (1997) 310-324.

[5] Vitanov, N. K. Upper Bounds on the Heat Transport in a Porous Layer. Physica $D, 136$ (2000), 322-339.

[6] Whitham, G. G. Linear and Nonlinear Waves, New York, Wiley, 1999.

[7] Martinov, N., N. Vitanov. On some Solutions of the Two-dimensional SineGordon Equation J. Phys A: Math. Gen, 25 (1992), L419-L426.

[8] Martinov, N., N. Vitanov. On the Correspondence between the Self-consistent 2D Poisson-Boltzmann Structures and the Sine-Gordon Waves. J. Phys A: Math. Gen, 25 (1992), L51-L56.

[9] Grimshaw, R. Nonlinear Waves in Fluids: Recent Advances and Modern Applications, Wien, Springer, 2005.

[10] Debnath, L. Nonlinear Water Waves, New York, Academic Press, 1994.

[11] Vitanov, N. K. Modified Method of Simplest Equation: Powerful Tool for Obtaining Exact and Approximate Traveling-wave Solutions of Nonlinear PDEs. Communications in Nonlinear Science and Numerical Simulations, 16 (2011), 1176-1185.

[12] Pedley, T. J. The Fluid Mechanics of Large Blood Vessels, Cambridge, Cambridge University Press, 1980.

[13] Mcdonald, D. A. Blood Flow in Arteries,London, Edward Arnold, 1974.

[14] Ku, D. N. Blood Flow in Arteries. Annual Review of Fluid Mechanics, 29 (1997), $399-434$. 
[15] Kudryashov, N. A. Simplest Equation Method to Look for Exact Solutions of Nonlinear Differential Equations. Chaos, Solitons 83 Fractals, 24 (2005), 12171231.

[16] Kudryashov, N. A., N. B. Loguinova. Extended Simplest Equation Method for Nonlinear Differential Equations. Applied Mathematics and Computation, 205 (2008), 396-402.

[17] Martinov, N., N. Vitanov. Running Wave Solutions of the Two-dimensional Sine-Gordon Equation. J. Phys A: Math. Gen., 25 (1992) 3609 -3613.

[18] Martinov, N., N. Vitanov. On the Solitary Waves in the Sine-Gordon Model of the Two-dimensional Josephson Junction. Zeitschrift für Physik B, 100 (1996), $129-135$.

[19] Vitanov, N. K. On Modified Method of Simplest Equation for Obtaining Exact and Approximate Solutions of Nonlinear PDEs: the Role of the Simplest Equation. Communications in Nonlinear Science and Numerical Simulation, 16 (2011), 42154231.

[20] Demiray, H. Non-linear Waves in a Fluid Filled Inhomogeneous Elastic Tube with Variable Radius. International Journal of Non-linear Mechanics, 43 (2008), 241-245.

[21] Saito, M., Y. Ikenaga, M. Matsukawa, Y. Watanabe, T. Asada, P.-Y. LAgreE. One-Dimensional Model for Propagation of a Pressure Wave in a Model of the Human Arterial Network: Comparison of Theoretical and Experimental Results. Journal of Biomechanical Engineering, 133 (2011), Article No. 121005.

[22] IL'IChev, A. T., Y.-B. Fu. Stability of Aneurism in a Fluid-filled Ellastic Membrane Tube. Acta Mechanica Sinica, 28 (2012), 1209-1218.

[23] Van Der Vosse, F. N., N. Stergiopoulos. Pulse Wave Propagation in the Arterial Tree. Annual Review of Fluid Mechanics, 43 (2011), 467-499.

[24] Gopalakrishnan, S. S., B. Pier, A. Biesheuvel. Dynamics of Pulsatile Flow through Model Abdominal Aortic Aneurysm. Journal of Fluid Mechanics, 758 (2014), 150-179.

[25] Misra, J. C., M. K. Patra. A Study of Solitary Waves in a Tapered Aorta by Using the Theory of Solitons. Computers $\&$ Mathematics with Applications, 54 (2007), 242-254.

[26] Fu, Y. B., A. T. IL'Ichev. Solitary Waves in Fluid-filled Elastic Tubes: Existence, Persistence, and the Role of Axial Displacement. IMA Journal of Applied Mathematics, 75 (2010), 257-268.

[27] Goldenvizer, A. L. Theory of Elastic Thin Shells, Oxford, Pergamon Press, 1961.

[28] Fung, Y. C. Biodynamics: Circulation, New York, Springer, 1981.

[29] Rudinger, G. Schock Waves in a Mathematical Model of Aortha. J. Appl. Mech., 37 (1970), 34-37. 
[30] Ivanov, P. Ch., L. A. N. Amaral, A. L. Goldberger, S. Havlin, M. G. Rosenblum, Z. R. Struzik, H. E. Stanley. Multifractality in Human Heartbeat Dynamics. Nature, 399 (1993), 461-465.

[31] Ivanov, P. Ch., M. G. Rosenblum, C. K. Peng, J. Mietus, S. Havlin, H. E. Stanley, A. L. Goldberger. Scaling Behaviour of Heartbeat Intervals Obtained by Wavelet-based Time-series Analysis. Nature, 383 (1996), 323-327.

[32] Vitanov, N. K., E. D. Yankoulova. Multifractal Analysis of the Long-range Correlations in the Cardiac Dynamics of Drosophila Melanogaster. Chaos Solitons E Fractals, 28 (2006), 768-775.

[33] Vitanov, N. K., N. P. Hoffmann, B. Wernitz. Nonlinear Time Series Analysis of Vibration Data from a Friction Brake: SSA, PCA, and MFDFA. Chaos, Solitons \& Fractals, 69 (2014), 90-99. 